\title{
Experiência do cliente no ponto de venda varejista: Desenvolvimento de uma escala de mensuração
}

\section{Customer experience at the point of retail sale: development of a measurement scale}

\author{
Roberto Rodrigues Ramos \\ Universidade Federal do Ceará \\ robertoramos1977@gmail.com \\ Francisco José da Costa \\ Unilab \\ Ingrid Mazza
}

Faculdade Paraíso - CE

Artigo Recebido em 19.07.2011. Revisado por pares em 12.11.2011. Recomendado em 12.11.2011 por Edson Roberto Scharf, Editor Responsável. Publicado em 30.06.2012. Organização responsável pelo periódico: FURB Universidade de Blumenau (www.furb.br/rn)

\begin{abstract}
Resumo
Este estudo tem por objetivo construir uma escala de mensuração da avaliação da experiência dos clientes no ponto de venda varejista. A verificação da literatura apontou a existência de algumas propostas de mensuração da avaliação da experiência de clientes no contexto varejista, porém verificaram-se limitações quando foram aplicadas no Brasil, o que indicou a necessidade de realização deste esforço. $\mathrm{O}$ estudo foi desenvolvido seguindo o conjunto de passos apontados na literatura especializada para construção de escalas. Foram tomadas duas amostras, uma para fins exploratórios (com 147 respondentes) e outra para fins confirmatórios (com 334 respondentes), e os procedimentos estatísticos usados viabilizaram a proposição de um instrumento com 19 itens, divididos em quatro dimensões (estrutura, sortimento, políticas e atendimento). Para assegurar a consistência da escala, foram verificadas as validades de conteúdo, de face, convergente, discriminante e de critério, além da confiabilidade da escala. Ao final do estudo, são dadas indicações de como a escala pode ser usada.
\end{abstract}

Palavras-chave: Varejo; Ponto de Venda; Escala.

\begin{abstract}
This study aims to propose a scale for measuring the evaluation retail customers experience in the instore context. The literature analysis indicated the existence of many different scales to measure the evaluation of the experience of customers in the retail context, but many limitations were found when these scales were used in Brazil, which indicated the need for implementation of this effort. The study was conducted following the series of steps suggested in the academic literature on scales construction. Two samples were taken, the first one for exploratory purposes (with 147 respondents) and another one for confirmatory purposes (with 334 respondents), and the statistical procedures used enabled the proposal of an instrument with 19 items, divided into 4 dimensions (structure, assortment, policies and interpersonal service). To ensure the consistency of the scale, content, face, convergent, discriminant and criterion validity were verified, in addition to the scale reliability. At the end of the study, indications of how the scale can be used are presented.
\end{abstract}

Key-Words: Retail; Store enviroment; Scale 


\section{Introdução}

O presente estudo mantém foco no varejo, com recorte específico na análise da avaliação pelos clientes de sua experiência no ponto de venda, com ênfase nos aspectos gerencialmente controláveis. Verificações exploratórias indicam a existência de um volume significativo de literatura sobre comportamento do consumidor no varejo e sobre sua experiência no ponto de venda, porém não se verificou homogeneidade nas abordagens. Adicionalmente, verificou-se uma lacuna na produção acadêmica brasileira sobre o tema, indicando assim a possibilidade de novas contribuições.

Os fatores da experiência de compra dentro da loja são bastante importantes em termos práticos e acadêmicos. A percepção que os clientes têm da loja, a preferência que demonstram por alguns atributos em detrimento de outros, e a conversão dessas preferências em vendas e em retorno às lojas são informações relevantes tanto para os gestores varejistas (pois contribuem para a administração eficiente das organizações) quanto para acadêmicos (pois aprimoram os conhecimentos de varejo e de comportamento do consumidor).

A ênfase deste estudo é a mensuração da avaliação por parte dos clientes de sua experiência. Estudos com preocupações em desenvolver escalas em marketing são poucos no Brasil (VIEIRA; SLONGO, 2007), e em nível internacional ainda constituem um grande desafio (NETEMEYER; BEARDEN; SHARMA, 2003). Alguns dos estudos pesquisados na fase de revisão de literatura (PARASURAMAN; ZEITHAML; BERRY, 1988; DABHOLKAR; THORPE; RENTZ, 1996; TERBLANCHE; BOSHOFF, 2002) foram direcionados para objetivos convergentes, porém em contex- tos e finalidades de aplicação diferentes dos propostos neste artigo.

Nestes termos, o objetivo central deste artigo é o seguinte: desenvolver uma escala de mensuração da avaliação, pelos clientes, de sua experiência nos pontos de vendas varejistas. $\mathrm{O}$ estudo se insere no contexto das pesquisas que buscam apresentar contribuições em nível temático (para as pesquisas de varejo e comportamento do consumidor) e metodológico (com a proposição de um instrumento consistentemente testado e apropriado para outros estudos).

Para atender ao objetivo enunciado, apresenta-se o estudo em três etapas, além desta introdução. A etapa seguinte apresenta a revisão de literatura sobre o assunto, com ênfase nas definições, importância e direcionamento da pesquisa para cada uma das dimensões selecionadas para análise; na sequiência, são demonstrados os procedimentos e as decisões referentes à metodologia utilizada para o desenvolvimento da escala, bem como os resultados da pesquisa, suas análises e descrição da amostra observada; e por último apresentam-se as considerações finais do trabalho, em conjunto com as suas limitações, implicações, e recomendações para pesquisas futuras.

\section{Referencial teórico}

Neste item apresenta-se o referencial teórico do estudo, com um debate preliminar sobre a experiência de consumidores no ambiente de loja do varejo, e em seguida com a exposição dos tópicos específicos da pesquisa direcionados ao desenvolvimento da escala.

\subsection{Experiência de consumidores no ambiente de loja do varejo}

O setor varejista, no Brasil e no mundo, tem demonstrado uma importância 
significativa no cenário econômico, comforme aponta Parente (2000) quando ressalta que, a partir de um ritmo forte de estabilização do varejo, as empresas da área aparecem constantemente na relação das maiores do Brasil (como exemplos têm-se o Grupo Pão de Açúcar, e as Casas Bahia). Como em qualquer outro tipo de organização, as políticas e estratégias mercadológicas dos varejistas são destinadas a conquistar e manter uma base de clientes sustentável e de longo prazo. A conseqüência dessa demanda de lealdade é a necessidade de um entendimento mais amplo sobre os variados fatores relacionados ao momento e local exatos onde e quando se dá o consumo.

A loja, também chamada de ponto de venda, é o espaço onde se dá a manifestação, pelo cliente, do comportamento de compra; e partir de então tem início um processo de avaliação e formação de atitudes para com o varejista (PARENTE, 2000). É no ponto de venda que se reúnem várias das atividades varejistas, sejam elas as decisões de marketing ou a interação entre provedor e consumidor.

Pode-se compreender que a análise do ponto de venda, e a experiência que o cliente tem com esse local, são ações que contribuem para atender a demanda acima citada. Tal experiência deve ser compreendida a partir da junção de um conjunto de componentes que influenciam o comportamento do consumidor (TERBLANCHE; BOSHOFF, 2006). Estudos indicam que a experiência vivida no ambiente de loja tem influência significativa na decisão de compra dos consumidores, inclusive nas compras por impulso. Nestes termos, a compreensão da experiência do consumidor no ponto de venda torna-se relevante para as organizações varejistas que têm como foco impactar de forma positiva na satisfação e na lealdade (COSTA et al., 2008).

Diversos trabalhos tiveram como tema central o ambiente de loja, especialmente na definição de que aspectos este se compõe (e. g. ZIMMER; GOLDEN, 1988; MCGOLDRICK; GREENLAND, 1994; TERBLANCHE; BOSHOFF, 2006), e na avaliação de como a percepção sobre esses aspectos influencia o cliente (e. g. BITNER, 1992; BAKER et al., 2002; COSTA et al., 2008).

Também foram observados esforços no sentido de mensurar esta experiência. Terblanche e Boshoff (2006), por exemplo, propuseram uma escala, considerando os elementos que fazem parte do contexto do ponto de venda são de duas naturezas: elementos físicos (sortimento, layout); e elementos sensoriais (aroma, cores, luzes). Já o estudo de Baker et. al. (2002) analisou como a percepção do cliente de vários fatores como o design, os funcionários e a música das lojas, influencia na sua decisão de compra. Donovan e Rossiter (1982) analisando aspectos relacionados ao prazer de comprar e seus estímulos, acreditam que um bom ambiente faz os consumidores passarem mais tempo na loja e consumirem mais.

$\mathrm{O}$ uso dos instrumentos desenvolvidos apresenta potenciais dificuldades para o contexto brasileiro. Por exemplo, Costa et al. (2008) replicaram o estudo de Terblanche e Boshoff (2006) no Brasil, e verificaram várias dificuldades conceituais e operacionais e nas dimensões analisadas.

\subsection{Recortes do estudo}

Através dos resultados da pesquisa bibliográfica, da exaustiva discussão entre os autores e de consultas a outros pesquisadores interessados no tema, ficou definido que a avaliação do comportamento do consumidor no ambiente do varejo seria inicialmente definida em cinco dimensões: layout, atmosfera, sortimento, políticas e atendimento.

A revisão bibliográfica dos conceitos dessas dimensões sugere a existência de correlações entre pares de dimensões, como, por exemplo, a relação entre atendi- 
mento e políticas, ou entre atmosfera e layout. Por outro lado, a literatura sugere que uma operacionalização em separado pode providenciar melhores condições para compreensão do objeto da pesquisa (a experiência dos clientes no ponto de venda), optou-se por uma abordagem inicialmente em separado, ficando qualquer decisão de agregação a ser tomada após o estudo empírico.

Sobre o layout, Parente (2000) o aponta como ligado a fatores que irão impactar na percepção inicial do consumidor, como a apresentação interna e a exposição de produtos. Baker et al.(2002) especificam mais essa definição quando afirmam que a configuração do layout é determinada pelos padrões de circulação e pela disposição dos equipamentos de exposição de produtos.

Baker et al.(2002) observam que o layout influencia a percepção do cliente em relação ao estilo e ao posicionamento de mercado de cada loja. Outros estudos ressaltam que este pode diminuir o tempo de busca do cliente, aumentar a eficiência, a consistência e a confiabilidade do serviço e facilitar a orientação do consumidor (CHASE; HAYES, 1991; BITNER, 1992). Com efeito, as decisões de layout devem motivar os consumidores a passar o tempo adequado na loja, alcançar maior produtividade do espaço e proporcionar um tráfego suave de consumidores, além de uma atmosfera prazerosa (PARENTE, 2000).

Já a atmosfera do ponto de venda relaciona-se, segundo Terblanche e Boshoff (2006), a todos os elementos que fazem parte de uma experiência de compra agradável, incluindo limpeza, decoração interior, dentre outros. Parente (2000) alega que o ambiente (ou atmosfera) é a personalidade da loja, incluindo os recursos visuais, cores, formas, sons, aromas, a decoração, e outros fatores que estimulam os sentidos do cliente e que vão construir seus sentimentos e emoções para com a loja. Alguns estudos consideram que o ambiente é essencialmente ligado à percepção dos atributos físicos do ponto de venda e dão pistas sobre a qualidade do serviço (BITNER, 1992; BAKER et al., 1992; DONOVAN; ROSSITER, 1982).

Como indica Parente (2000), a imagem que os clientes têm da loja está intimamente ligada à atmosfera que a loja consegue desenvolver. Conforme apontam vários autores, há evidências de que o comportamento e atitude dos consumidores varejistas pode ser influenciada pelo ambiente (DONOVAN; ROSSITER, 1982; DARDEN; ERDEM; DARDEN, 1983; RIDGEWAY; DAWSON; BLOCH, 1989). Nestes termos, entende-se que os atributos físicos de uma loja, sua aparência e apresentação se combinam para dessempenhar um papel muito importante na oferta de produtos de uma loja. Não sem razão, muitas pesquisas fizeram avanços no entendimento dos argumentos conceituais que suportam a ligação entre qualidade do serviço e a percepção do ambiente da loja como um todo (BAKER 1987; BITNER 1992; GREENLAND; MCGOIDRICK 1994). É relevante o trabalho de Titus e Everett (1995), que mostra como o layout influencia as expectativas dos consumidores de uma movimentação mais eficiente dentro da loja. Vários outros trabalhos concluem que os atributos físicos, conceito que inclui o layout, são bastante valorizados, pois geram conveniência para o consumidor (GUTMAN; $\quad$ ALDEN, 1985; MAZURSKY; JACOBY, 1985; HUMMEL; SAVITT, 1988).

O sortimento refere-se ao mix de produtos apresentados na loja, envolvendo variedade de categorias e de marcas (GREENLEY; SHIPLEY, 1988), e é um dos mais conhecidos componentes do mix de marketing convencional do varejo 
(HASTY; REARDRON, 1997). Segundo Terblanche e Boshoff (2006), o sortimento está relacionado diretamente com 0 número de produtos ofertados e suas categorias, ou seja, uma relação entre amplitude e profundidade da linha de produtos ofertados pelo varejista.

É possível avaliar o sortimento tanto na ótica do cliente quando do varejista. Sob a ótica do cliente, a variedade e a seleção de diferentes tipos de produtos é uma das mais complexas expectativas a serem satisfeitas por parte do ofertante, pois o produto constitui a finalidade primária da decisão de consumo em determinada loja. Já pela ótica do varejista, Parente (2000) sugere que uma seleção adequada da linha de produtos confere um aspecto de diferenciação, observando ainda que há um desafio comstante no sentido das empresas varejistas encontrarem um equilíbrio ente variedade excessiva e variedade incompleta.

Alguns trabalhos, como os de Cox (1970) e Curhan (1972), estudaram o sortimento medindo seus efeitos nas prateleiras de supermercados, o que levou a vários outros estudos sobre a seleção de itens, além da relação entre o número de produtos e suas categorias (CORSTJENS; DOYLE, 1981; BULTEZ; NAERT, 1988). Há trabalhos que relacionam os efeitos do sortimento, incluindo o efeito da disponibilidade e variedade de itens nas intenções de compra dos consumidores. Koelemeijer e Oppewal (1999; p. 341), por exemplo, encontraram evidências empíricas que "o aumento na variedade atrai compras adicionais proporcionalmente à atratividade dos itens adicionados".

As políticas das lojas estão relacionadas a como o varejista gerencia as devoluções ou trocas de produtos, horários de funcionamento, formas de pagamento e reclamações (DICKSON; ALBAUM, 1977; $\quad$ WESTBROOK, 1981; MAZURSKY; JACOBY, 1985). Parente (2000) realça a importância destes aspectos quando defende que o varejista deve oferecer suporte para facilitar o consumidor no seu processo de compra, fornecendo informações sobre mercadorias disponíveis, preços, condições de crédito e outras relativas à loja e aos produtos.

Dabholkar, Thorpe e Rentz (1996) acessam o tema no contexto de avaliação da qualidade do serviço no varejo, através de itens semelhantes aos usados nesse trabalho, tais como formas de pagamento, trocas e devoluções, horário de funcionamento, refletindo o que os autores chamaram somente por 'políticas'. Por outro lado, no mesmo trabalho, propuseram outra dimensão chamada 'resolução de problemas' que inclui aspectos relacionados à reclamação por parte dos consumidores (DABHOLKAR; THORPE; RENTZ, 1996). Neste trabalho as duas estão reunidas em uma única dimensão chamada políticas.

Atendimento é uma parte do serviço geral, e inclui o tratamento dos clientes e a atenção pessoal dispensada pelos funcionários (BAKER et al., 2002). Parente (2000) afirma que o atendimento engloba todas as atividades que apresentam um relacionamento pessoal entre $\mathrm{o}$ varejista e o consumidor. Para o autor, todos os funcionários que têm qualquer interação com os consumidores dessempenham alguma atividade de venda.

Quando o atendimento é dessempenhado de forma adequada, o varejista consegue desenvolver relações de longo prazo, para manter a fidelidade do consumidor. Independentemente do tipo de loja, a qualidade do relacionamento pessoal com os consumidores será um fator fundamental de diferenciação e de vantagem competitiva. (PARENTE, 2000). A importância do atendimento foi observada inclusive por Parasuraman, Zeithaml e Berry (1988), em seu clássico estudo sobre qualidade de serviços, com a indicação de que a quantidade e a aparência dos funcionários em uma loja de varejo são 
percebidas pelo consumidor como um sinal

\begin{tabular}{|c|c|}
\hline $\begin{array}{l}\text { Passo } 1 \text { - Especificação do domínio do } \\
\text { construto }\end{array}$ & $\begin{array}{l}\text { Passo } 2 \text { - Atividades de geração de itens e } \\
\text { validação de face e conteúdo }\end{array}$ \\
\hline$\downarrow$ & \\
\hline Passo 3 - Decisões sobre as respostas & $\begin{array}{l}\text { Passo } 4 \text { - Construção do instrumento de } \\
\text { pesquisa }\end{array}$ \\
\hline \multicolumn{2}{|l|}{$\downarrow$} \\
\hline $\begin{array}{l}\text { Passo } 5 \text { - Primeira atividade de amostragem } \\
\text { e procedimentos de limpeza da escala }\end{array}$ & $\begin{array}{l}\text { Passo 6 - Segunda atividade de amostragem } \\
\text { e procedimentos de limpeza da escala }\end{array}$ \\
\hline \multicolumn{2}{|l|}{$\nabla$} \\
\hline $\begin{array}{l}\text { Passo } 7-\text { Análise de validade e de } \\
\text { confiabilidade da escala final }\end{array}$ & $\begin{array}{l}\text { Passo } 8 \text { - Desenvolvimento de normas e } \\
\text { recomendações de uso e interpretação }\end{array}$ \\
\hline
\end{tabular}

Figura 1 - Passos do desenvolvimento da escala

Fonte: Elaborado pelos autores

varejo são percebidas pelo consumidor como um sinal tangível da qualidade do serviço.

Pesquisas indicam a relevância do atendimento no esforço relacional das empresas. Grewal e Sharma (1991), por exemplo, mostraram que a equipe de vendas tem um papel crítico na influência do comportamento e satisfação dos consumidores. Baker et al. (2002) mostra que interações positivas entre consumi-dores e funcionários, como o fato de reconhecer um cliente quando ele entra na loja, por exemplo, podem influenciar a percepção de qualidade do consumidor (BAKER et al, 2002). Já Costa et al. (2008) apresentaram indicações de que o atendimento se mostra como um componente relevante na formação da confiança e da lealdade de clientes varejistas, a partir da mediação da satisfação.

\section{Desenvolvimento da escala}

De acordo com a literatura que trata do desenvolvimento de escalas em marketing (CHURCHILL, 1979; DEVELLIS, 1991; NETEMEYER; BEARDEN; SHARMA, 2003), são delineáveis oito passos essenciais para o desenvolvimento de uma escala em ciências sociais. Este conjunto de passos está descrito na Figura 1.

A seguir são descritos de forma detalhada cada um desses passos na presente pesquisa. Para o melhor aproveitamento do espaço no artigo, a sequiência em que os passos serão apresentados se dará da seguinte forma: de 1 a 6 os passos são apresentados juntos, em duplas, seguindo a sequência numérica. Já os últimos passos, 7 e 8 , são apresentados em separado, devido as suas particularidades, tamanho e grande valor para o estudo.

\subsection{Passos 1 e 2 - conceituação em geração de itens}


Em relação ao passo 1, que diz respeito às especificações dos domínios das dimensões, inicialmente foi feita uma pesquisa na literatura disponível em bases de dados científicas, o que permitiu a visualização de como se encontrava o tema em termos de pesquisas acadêmicas. Os resultados desta fase viabilizaram a conceituação e contextualização das dimensões de análise, conforme mostrado no item 2 deste estudo.

Como mencionado anteriormente, todas as dimensões avaliadas para esse estudo já haviam sido objeto de estudos anteriores, com mudanças no enfoque, no contexto ou na reunião das dimensões relacionadas. Assim, a geração de itens para a proposta dessa nova escala foi baseada nestes estudos, a partir dos quais foram gerados inicialmente 62 itens distribuídos nas cinco dimensões de análise previamente delineadas. O critério fundamental para seleção destes itens era que mantivessem, inicialmente, adequação semântica às dimensões, e que mantivessem com estas uma relação claramente refletiva, como forma de viabilizar as operacionalizações segundo os modelos da teoria clássica da mensuração e evitar os riscos de má especificação (JARVIS; MACKENZIE; PODSAKOFF, 2003).

De posse destes itens, buscou-se inicialmente providenciar a validade de conteúdo, na qual se analisa o grau de relevância e representatividade dos itens perante a dimensão para uma avaliação especifica; e ainda a validação de face, a partir da qual se pode obter um nível de praticidade, pertinência, e representatividade de um conjunto de itens em relação à dimensão a ser medida (NETEMEYER; BEARDEN; SHARMA, 2003).

Assim, o conjunto de itens foi submetido a outros pesquisadores e interessados no tema, com as devidas explicações do propósito e dos conceitos associados para o conjunto de itens. Este procedimento gerou algumas correções de enunciado para simplificação de entendimento, além de indicações de itens redundantes, e de alternativas de mudança na adesão dos itens às dimensões. Após este procedimento, os itens foram reavaliados com base nas sugestões e críticas, ficando 54 itens subdivididos entre as 5 primeiras dimensões pesquisadas, sendo 12 itens para layout, 11 para atmosfera, 9 para sortimento e 11 para políticas e 11 para atendimento.

\subsection{Passos 3 e 4 - decisões de resposta e instrumento}

Quanto às decisões pertinentes às respostas dos itens, foi possível observar na literatura pesquisada sobre a escala, a disponibilidade de várias alternativas (Likert, diferencial semântico...). Considerando os estudos desenvolvidos com propósitos semelhantes ao deste, optou-se inicialmente pela utilização da escala de Likert, apresentada com 7 pontos, sempre com 1 indicando discordância total, e 7 concordância total.

Para a construção do instrumento de pesquisa, decidiu-se pela disposição dos 54 itens da dimensão em três blocos, como forma de evitar dificuldades de respostas. Foram reunidos os itens de atendimento e layout e os de sortimento e de políticas, ficando os itens de atmosfera em um bloco único. $\mathrm{Na}$ apresentação, os itens foram dispostos de modo a evitar que dois itens de uma mesma dimensão ficassem em seqüência.

Além das questões sobre as dimensões, foram acrescentadas no questionário ainda quatro questões sobre dados demográficos, além de uma sobre a freqüência de compra nas lojas. Ao final, o instrumento de pesquisa foi formatado e foi possível sua apresentação em duas páginas. Uma primeira versão foi apresentada a 
outros pesquisadores e foi realizado um pré-teste com vinte respondentes. Após os devidos ajustes, o instrumento foi consolidado para sua aplicação em campo.

\subsection{Passos 5 e 6 - amostragem e limpeza}

Após a consolidação do questionário, um levantamento foi realizado junto a 147 respondentes de uma cidade de grande porte da região Nordeste do Brasil, sendo 76 pessoas abordadas aleatoriamente pelos pesquisadores, acessadas por conveniência, e 71 respondentes abordados diretamente em três diferentes lojas (uma loja de CDs e DVDs, uma de variedades, e outra de produtos de informática). A aplicação dos questionários foi realizada nos meses de julho e agosto de 2008. A média de tempo para a finalização do questionário foi de aproximadamente quinze minutos, e a abordagem foi por conveniência e acessibilidade. Esta primeira amostragem tem por finalidade gerar dados para uma avaliação exploratória da estrutura da escala.

A amostra foi formada em sua maioria por pessoas com escolaridade alta, $77,4 \%$ possuindo de nível superior (completo ou incompleto), com uma grande concentração de jovens $(39,7 \%$ com idade até 25 anos; e $30,8 \%$ entre 25 e 30 anos), e uma predominância de sexo masculino (62,3\% dos respondentes).

Os dados coletados foram consolidados, e foram procedidas às análises iniciais de dados perdidos (missing values) e valores extremos (outliers), não tendo sido observada qualquer demanda de procedimento de exclusão de item ou entrada de dados. Em seguida, as variáveis das dimensões foram submetidas a um conjunto de verificações, em três diferentes momentos: primeiramente era aplicada técnica de análise fatorial exploratória AFE; em seguida, era analisada a matriz de correlação bivariada; por fim, era proce- dida a verificação da confiabilidade por meio do coeficiente alpha de Cronbach. Todos os procedimentos foram realizados por dimensão, e foram desenvolvidos com suporte do software SPSS, versão 15. Para cada uma das verificações era analisada a consistência das medidas, e verificada a possibilidade de exclusão de itens. Os resultados estão expostos a seguir:

- Para a dimensão 'layout' foram retirados seis itens, restando outros seis itens ao final. O escore fatorial mínimo, após as retiradas dos itens, foi de 0,716 , com uma variância extraída de 57,63\%. A correlação mínima foi de 0,328, e o alpha de Cronbach foi de 0,851

- Para a dimensão 'atmosfera' foram excluídas cinco variáveis, restando seis itens ao final. $\mathrm{O}$ escore fatorial mínimo, após as retiradas dos itens, foi de 0,738 e a variância extraída foi de $63,68 \%$. A correlação mínima foi de 0,470, e o alpha de Cronbach foi de 0,885

- Para a dimensão 'sortimento' foram retirados três itens, restando seis itens ao final. $\mathrm{O}$ escore fatorial mínimo, após as retiradas dos itens, foi de 0,727 e a variância extraída foi de $62,13 \%$. A correlação mínima foi de 0,727 e o alpha de Cronbach foi de 0,874;

- Para a dimensão 'políticas', foi necessária a exclusão de cinco itens, restando seis ao final das análises. $\mathrm{O}$ escore fatorial mínimo, após as retiradas dos itens, foi de 0,678 e observou-se uma variância extraída de 59,26\%. A correlação mínima foi de 0,382 , e o alpha de Cronbach foi de 0,849 .

- Para a dimensão 'atendimento' foram excluídas cinco variáveis, restando seis itens ao final. $\mathrm{Na}$ análise fatorial o escore fatorial mínimo, após as retiradas dos itens, foi de 0,834 e observou-se 
uma variância extraída de $75,25 \%$. A correlação mínima foi de 0,645 , e o alpha de Cronbach foi de 0,931;

Após estas análises, restaram 31 itens das dimensões da escala. De posse deste resultado, um novo questionário foi construído, para uma segunda aplicação. A escala de respostas foi modificada de 7 para 5 pontos, visando uma maior simplificação do processo de resposta, e o ordenamento e divisão dos itens em blocos seguiu a mesma lógica do instrumento usado na primeira aplicação. Também foram adicionadas variáveis para mensurar o construto satisfação, com seis itens extraídos Bonfim et al. (2008), e Soares (2008), o que permitiu a análise de validade de critério.

Após uma nova rodada de avaliação do questionário junto a especialistas e pesquisadores, o instrumento de coleta foi consolidado, e foi realizada a segunda entrada em campo (na mesma cidade), com a aplicação de 334 questionários, junto a clientes de 20 lojas, de três diferentes segmentos (produtos infantis - 38,9\%, produtos de informática - $29,9 \%$, e moda praia-31,2\%). A coleta foi procedida com a colaboração dos funcionários das lojas. Esta segunda amostragem tem finalidade gerar dados para uma avaliação confirmatória da estrutura da escala.

Após uma análise preliminar, foram descartados 84 questionários que estavam mal respondidos, incompletos ou inadequados para o uso, restando válidos 250 questionários. Também foi procedida a exploração preliminar, com a verificação de valores faltantes (missing values), tendo sido descartadas 6 entradas de dados devido a esse critério, assim como da existência de valores atípicos (outliers) nos itens das dimensões, o que conduziu à retirada de mais 8 entradas. Ao final, restaram 236 entradas válidas.

$\mathrm{Na}$ amostra, houve um maior equi- líbrio no gênero dos respondentes, com $53,8 \%$ de mulheres e $46,2 \%$ de homens. A maioria dos respondentes continuou na faixa de até $25 \operatorname{anos}(38,3 \%)$ e entre 25 e 30 anos (26\%). Analisando a renda familiar mensal, percebeu-se uma boa representatividade de todas as faixas, com destaque para a faixa de até $\mathrm{R} \$ 1.000,00$, com $30,6 \%$ dos respondentes, e a faixa acima de $\mathrm{R} \$ 3.000,00$, com 28,4\% dos pesquisados. A frequiência de compra mostrou que uma parte representativa $(38,6 \%)$ dos entrevistados é constituída por clientes que compram com uma freqüência pelo menos mensal nas lojas, e uma porção igualmente significativa indicou $36,1 \%$ consumir esporadicamente. Os resultados das características das amostras se aproximam da realidade (exploratóriamente) verificada no universo dos consumidores das lojas dos segmentos varejistas pesquisados.

Novamente aqui foram procedidas exploratoriamente as análises fatorial, de correlação e de confiabilidade, não tendo sido observadas grandes discrepâncias em relação ao resultado da primeira amostra. Considerando ainda que a segunda etapa do trabalho demanda uma análise fatorial confirmatória, optou-se por omitir os detalhes da análise exploratória e seguir para as análises subseqüentes de validade.

\subsection{Passo 7 - validade e confiabilidade da escala}

Este item foi dividido em três partes: inicialmente apresentam-se os resultados da análise fatorial confirmatória; em seguida procede-se à análise de validade de dimensão e de critério; ao final apresentam-se os resultados de confiabilidade.

\subsubsection{Análise fatorial confirmatória}

Para a análise fatorial confirmatória, inicialmente foi necessário definir aspectos associados à avaliação das dimensões, 
especialmente nos índices de ajustamento. Verificou-se que os índices e seus valores de referência variam na literatura especializada, tendo sido adotados os seguintes, com os respectivos pontos de corte: a estatística qui-quadrado $\left(\chi^{2}\right)$, que

Tabela 1 - Medidas finais de ajuste deve ser a mais baixa possível, e sua divisão pelo número de graus de liberdade é boa se menor que 3; a raiz do erro quadrático médio aproximado (RMSEA), que deve ser menor que 0,08 ; o Índice de Adequação do Ajustamento ao final.

\begin{tabular}{|c|c|c|c|c|c|}
\hline \multirow{2}{*}{ MEDIDA } & \multicolumn{5}{|c|}{ DIMENSÃO SOB ANÁLISE } \\
\hline & Estrutura & Sortimento & Políticas & Atendimento & Satisfação \\
\hline Confiabilidade composta & 0,807 & 0,828 & 0,798 & 0,868 & 0,909 \\
\hline Variância extraída & 0,512 & 0,500 & 0,447 & 0,569 & 0,626 \\
\hline Média dos escores & 0,714 & 0,694 & 0,661 & 0,752 & 0,789 \\
\hline Menor critical ratio* & 8,737 & 6,277 & 6,241 & 9,525 & 10,741 \\
\hline Qui-quadrado $\left(\chi^{2}\right)$ & 2,090 & 7,534 & 7,619 & 8,230 & 13,121 \\
\hline Graus de liberdade (gl) & 2 & 5 & 5 & 5 & 9 \\
\hline$\chi^{2} / \mathrm{gl}$ & 1,045 & 1,507 & 1,524 & 1,646 & 1,458 \\
\hline$p$ value & 0,352 & 0,184 & 0,178 & 0,144 & 0,157 \\
\hline GFI & 0,996 & 0,988 & 0,987 & 0,986 & 0,982 \\
\hline CFI & 1,000 & 0,994 & 0,992 & 0,994 & 0,995 \\
\hline RMSEA & 0,014 & 0,046 & 0,047 & 0,052 & 0,044 \\
\hline Alpha final & 0,800 & 0,809 & 0,788 & 0,864 & 0,909 \\
\hline
\end{tabular}

Notas: *Todos valores significantes a $\mathrm{p}<0,001$

Fonte: dados da pesquisa

(GFI), e o Índice de Ajuste Comparativo (CFI), que são bons se maiores que 0,9 (HAIR et al, 2005).

Os procedimentos foram realizados com suporte do software AMOS (versão 7), e as dimensões foram avaliadas inicialmente a partir dos conjuntos de variáveis provenientes da primeira aplicação, por dimensão. Para cada dimensão eram extraídos os índices de ajustamento, juntamente com a confiabilidade composta, a variância extraída, a média dos escores fatoriais, e o menor critical ratio. Os principais resultados foram os seguintes: para as dimensões de, 'atmosfera', 'sortimento', 'políticas' e 'atendimento' um ajuste adequado somente foi possível com a exclusão de uma variável em cada uma das dimensões, restando cinco em cada, ao final; para a dimensão 'layout' foi necessária a exclusão de duas variáveis, restando quatro
Após estes procedimentos foi retirada a correlação entre os pares de dimensões, como forma de verificar possíveis colinearidades. Após este passo, verificou-se que as dimensões 'layout' e 'atmosfera' apresentaram uma correlação de 0,94 (o que é indicativo de colinearidade), o que indicou a necessidade de avaliação da possibilidade de reunião das variáveis em uma só dimensão. Avaliou-se o sentido e os enunciados de cada um dos itens, tendo-se verificado que estes guardavam entre si similaridades, viabilizando a reunião. Assim, as quatro variáveis de 'layout' foram avaliadas juntamente com as cinco variáveis finais de 'atmosfera', e os procedimentos de ajuste conduziram a uma nova dimensão, com três variáveis de 'layout', e uma de 'atmosfera'. O sentido dos enunciados desta nova dimensão possibilitou que a 
nova dimensão fosse identificada por 'estrutura'.

O mesmo procedimento de extração de medidas foi aplicado ao conjunto de variáveis de satisfação, com a finalidade de dar consistência a este construto, que serviu de base para análise de validade de critério. Nesta análise, não foi necessária a exclusão de nenhuma das seis variáveis. As medidas finais das quatro dimensões que restaram, juntamente com as medidas de satisfação, estão expostas na Tabela 1 .

\subsubsection{Validades convergente, discriminante e de critério}

De posse destes resultados, que asseguram que as variáveis medem adequadamente as dimensões sob análise, foi possível averiguar as validades convergente, discriminante e de critério. A primeira (validade convergente) avalia até que ponto as variáveis da escala se correlacionam positivamente com outras medidas da mesma dimensão (DEVELLIS, 1991), e pode ser acessada a partir da significância das cargas fatoriais dos indicadores, pela análise dos t-values (no software AMOS pela análise dos critical ratios - CR), sendo que valores superiores a 1,96 (ou significativos a $p<0,05$ ) indicam resultados adequados.

Conforme é possível verificar na Tabela 1, o menor valor de critical ratio por dimensão foi de 6,241 (dimensão políticas), bem superior ao valor mínimo de 1,96 , e todos os valores foram significativos a $\mathrm{p}<0,001$. Nestes termos, tem-se assegurada a validade convergente para cada dimensão.

Já a validade discriminante indica o quanto a medida de uma dimensão não se correlaciona com outras dimensões as quais deve diferir (DEVELLIS, 1991). Nestes termos, a validade discriminante é assegurada quando a medida de uma dimensão não apresentar correlação muito elevada com as outras medidas. Para este estudo, a ênfase foi restrita às quatro dimensões sob análise, que por hipóteses são distintas entre si. Assim, foi procedida a comparação entre a variância extraída em cada dimensão e a variância compartilhada com as demais dimensões (medida pelo quadrado do coeficiente de correlação de Pearson), conforme recomendam Hair et al. (2005).

Conforme é possível verificar na Tabela 2, os valores de variância compar-tilhada são menores que os valores de variância extraída nas dimensões, havendo, no entanto, duas exceções, a variância compartilhada de atendimento e políticas ficou acima das variâncias extraídas e a de sortimento e políticas ficou acima das variâncias extraídas (a análise do sentido das dimensões justifica esta situação, pois os aspectos relacionados ao atendimento e ao sortimento da loja associam-se às suas políticas). Por outro lado, a correlação entre estas dimensões foi menor que 0,9 , o que indica a inexistência de colinearidade entre as variáveis, indicando assim que estas três dimensões são distintas, porém apresentam forte correlação. Nestes termos, é possível compreender que as dimensões são validade discriminante.

Tabela 2 - Variâncias extraídas e compartilhadas

Fonte: dados da pesquisa

\begin{tabular}{|l|c|c|c|c|}
\hline & $\begin{array}{c}\text { Estru- } \\
\text { tura }\end{array}$ & $\begin{array}{c}\text { Sorti- } \\
\text { mento }\end{array}$ & $\begin{array}{c}\text { Pol. } \\
\text { Aten- } \\
\text { dimento }\end{array}$ & $\begin{array}{c}\text { Aten- } \\
\text { dimento }\end{array}$ \\
\hline $\begin{array}{l}\text { Estru- } \\
\text { tura }\end{array}$ & 0,512 & & & \\
\hline $\begin{array}{l}\text { Sorti- } \\
\text { mento }\end{array}$ & 0,232 & 0,500 & & 0,748 \\
\hline $\begin{array}{l}\text { Pol. } \\
\text { Atendi- } \\
\text { mento }\end{array}$ & 0,404 & 0,594 & 0,447 & \\
\hline $\begin{array}{l}\text { Atendi- } \\
\text { mento }\end{array}$ & 0,276 & 0,349 & 0,748 & 0,569 \\
\hline
\end{tabular}

Por fim, foi averiguada a validade 
de critério, que se refere ao grau o quanto uma medida (co)varia com outras previamente validadas, de modo que, se a variação for simultânea, temos a validade de critério simultâneo, e se for tomada em momentos distintos, temos a validade de critério preditivo (DEVELLIS, 1991). Assim, a satisfação foi definida como critério para avaliação simultânea (ver item 3.3), pois apresentou evidências de relação com as dimensões sob análise em outros estudos (TERBLANCHE; BOSHOFF, 2006; BONFIM et al., 2008). Na verificação da correlação de 'satisfação' com as diferentes dimensões, os resultados indicaram valores entre intermediários e elevados (com estrutura - 0,599; com sortimento 0,737 ; com políticas $-0,881$; com atendimento - 0,804), o que dá evidência de validade de critério simultâneo.

\subsubsection{Confiabilidade}

Com os resultados consolidados da análise fatorial confirmatória e na análise de validade, foi possível também consolidar as indicações de confiabilidade. Conforme indica a literatura especializada, uma medida relevante para indicação de confiabilidade é o coeficiente alpha de Cronbach, que foi extraído ao final da operacionalização dos itens em cada dimensão. Conforme é possível verificar na Tabela 1, a confiabilidade foi assegurada uma vez que o menor valor de alpha en- contrado foi de 0,788 (dimensão políticas).

Alternativamente, também é possível verificar a confiabilidade de dimensões a partir da confiabilidade composta, que consiste em uma medida de consistência interna dos indicadores de uma dada dimensão, e que descreve o quanto esses indicam a dimensão latente em comum, e da variância extraída, que reflete a quantidade geral de variação dos indicadores que é explicada pela dimensão latente (HAIR et al., 2005, p. 489). Seguindo as recomendações da literatura especializada, assegura-se confiabilidade quando a confiabilidade composta é maior que 0,7 , e a variância extraída é maior que 0,5. Conforme é possível verificar na Tabela 1, a menor confiabilidade composta foi de 0,798 , e a menor variância extraída foi de 0,447 (ambos da dimensão políticas), contudo nessa dimensão todas as outras medidas foram consistentes. Nestes termos, também aqui, pode-se aceitar que há evidências de confiabilidade na mensuração das dimensões.

Consolidada a avaliação de validade e de confiabilidade, a escala final ficou com um total de 19 itens, dispostos em 4 dimensões. Os itens de cada dimensão e as medidas dos escores fatoriais, das médias e dos desvios padrão da segunda amostra, estão na Tabela 3 .

Tabela 3 - itens da escala

\begin{tabular}{|c|c|c|c|}
\hline Dimensões e itens da escala & Escore & Média & Desvio \\
\hline \multicolumn{4}{|c|}{$\begin{array}{ll}\text { Estrutura } \\
\end{array}$} \\
\hline A estrutura física da loja é atrativa & 0,801 & 4,07 & 1,01 \\
\hline A estrutura geral da loja é bem organizada & 0,812 & 4,10 & 0,92 \\
\hline As instalações físicas são visualmente atraentes & 0,765 & 3,94 & 1,02 \\
\hline O ambiente é propício para fazer compras & 0,803 & 4,35 & 0,75 \\
\hline \multicolumn{4}{|c|}{ Sortimento } \\
\hline Há uma boa variedade de marcas & 0,774 & 4,22 & 0,84 \\
\hline A loja possui diferentes linhas de produtos & 0,837 & 4,31 & 0,81 \\
\hline Sempre encontro na loja os produtos que procuro & 0,564 & 3,86 & 1,03 \\
\hline Encontro na loja diversas opções de produtos & 0,847 & 4,20 & 0,85 \\
\hline
\end{tabular}


A loja disponibiliza produtos das melhores marcas 0,785

\section{Política de Atendimento}

Há facilidade em eventuais necessidades de trocas e devoluções

A loja sabe lidar com as reclamações de forma justa

Consigo ter acesso aos gerentes

Sinto-me respeitado como consumidor nesta loja

A quantidade de atendentes é suficiente para a demanda dos clientes Atendimento

O pessoal de atendimento da loja é bastante prestativo

O pessoal de atendimento dá uma boa atenção aos clientes

Os atendentes são amigáveis

O pessoal de atendimento é interessado em resolver problemas

Os atendentes são corteses/delicados com os clientes

\begin{tabular}{l|l|l|}
\hline 0,725 & 3,97 & 1,02
\end{tabular}

\begin{tabular}{l|l|l}
0,819 & 4,04 & 0,92
\end{tabular}

\begin{tabular}{l|l|l}
0,760 & 4,17 & 1,02
\end{tabular}

\begin{tabular}{l|l|l}
0,776 & 4,36 & 0,79
\end{tabular}

\begin{tabular}{l|l|l}
0,623 & 3,95 & 1,03
\end{tabular}

Fonte: dados da pesquisa

\subsection{Passo 8 - recomendações}

O último passo do desenvolvimento da escala consiste no estabelecimento de recomendações de uso. Para este estudo, tem-se o seguinte:

- Sobre a forma de apresentação, recomenda-se o uso da escala de Likert de 7 pontos, que providencia mais informações por item, e facilita o alcance de uma condição de normalidade (fundamental para algumas técnicas estatísticas). Alternativamente, a escala também pode ser aplicada na escala de 5 pontos, o que facilita a coleta junto aos respondentes;

- As dimensões de análise medem ao final a avaliação da experiência do cliente no ponto de venda varejista, porém tratam de dimensões diferentes entre si. Adicionalmente, o número final de itens por dimensão foi distinto, variando entre 4 ou 5 . Nestes termos, é possível encontrar uma medida geral de cada dimensão a partir da extração das médias dos escores de cada entrada em uma mesma dimensão. Este procedimento gera sempre medidas no intervalo da escala, mas tem a desvantagem de reduzir a variabilidade comparativamente a cada variável separadamente (BAGOZZI;
- EDWARDS, 1998). Acredita-se, por outro lado, que a manutenção da escala na pontu-ação original (de 1 a 7 ou de 1 a 5) ajuda na interpretação dos resultados;

- Na literatura especializada não foram encontradas indicações com boas justificativas de interpretação de estatísticas descritivas em escalas de intervalo, como é o caso deste

Quadro 1 - Recomendações de análise

Fonte: Elaborado pelos autores

\begin{tabular}{|c|c|c|c|}
\hline \multirow{2}{*}{ Medida } & \multirow{2}{*}{ Nível } & \multicolumn{2}{|c|}{$\begin{array}{l}\text { Número de } \\
\text { pontos }\end{array}$} \\
\hline & & $\begin{array}{c}5 \\
\text { pontos }\end{array}$ & $\begin{array}{c}7 \\
\text { pontos }\end{array}$ \\
\hline \multirow{3}{*}{$\begin{array}{c}\text { Critério para } \\
\text { média }\end{array}$} & Baixo & $\begin{array}{l}\text { Até } \\
2,99\end{array}$ & $\begin{array}{l}\text { Até } \\
3,99\end{array}$ \\
\hline & $\begin{array}{l}\text { Interme- } \\
\text { diário }\end{array}$ & $\begin{array}{l}\text { De } 3,0 \\
\text { a } 3,99\end{array}$ & $\begin{array}{l}\text { De } 4,00 \\
\text { a } 5,49\end{array}$ \\
\hline & Elevado & $\begin{array}{l}\text { A partir } \\
\text { de } 4,00\end{array}$ & $\begin{array}{l}\text { A partir } \\
\text { de } 5,50\end{array}$ \\
\hline \multirow{3}{*}{$\begin{array}{c}\text { Critério para } \\
\text { o desvio } \\
\text { padrão }\end{array}$} & Baixo & $\begin{array}{l}\text { Até } \\
0,80\end{array}$ & $\begin{array}{l}\text { Até } \\
1,00\end{array}$ \\
\hline & $\begin{array}{l}\text { Interme- } \\
\text { diário }\end{array}$ & $\begin{array}{l}\text { De } 0,81 \\
\text { a } 1,00\end{array}$ & $\begin{array}{l}\text { De } 1,01 \\
\text { até } 1,50\end{array}$ \\
\hline & Elevado & $\begin{array}{l}\text { Acima } \\
\text { de } 1,01\end{array}$ & $\begin{array}{l}\text { Acima } \\
\text { de } 1,51\end{array}$ \\
\hline
\end{tabular}

- trabalho, porém a experiência dos autores indica como razoáveis os critérios para avaliação de medidas de 
média e desvio padrão apontados no Quadro 1;

- Para a aplicação da escala, recomendase que os 19 itens sejam dispostos em ordem aleatória, de preferência separados em dois blocos, como forma de evitar com que o respondente proceda a associações entre as variáveis e venha com isto a enviesar as respostas.

\section{Considerações finais}

Este estudo buscou contribuir na análise da avaliação dos clientes de sua experiência no ponto de venda varejista, tendo em vista que a área de varejo, especificamente o que diz respeito ao ponto de venda, tem sido o foco de inúmeros estudos acadêmicos e continua sendo um tema relevante em marketing. Entende-se ter alcançado o objetivo principal do trabalho, que foi o de desenvolver uma escala de mensuração, o que pode ser observado a partir da descrição das diversas etapas da construção e validação da escala. As características psicométricas de uma boa escala foram todas atingidas, conforme sintetizado a seguir:

- Com relação à validade de conteúdo e face, foram verificadas indicações consistentes de validade nos primeiros passos da pesquisa, conforme relatado no item 3.1;

- A análise de validade de construto, nas formas de validade convergente $\mathrm{e}$ discriminante, foi confirmada tomando por base os resultados da análise fatorial confirmatória, a partir da verificação dos índices de ajustamento propostos na literatura especializada, conforme expôs o item 3.4.2;

- No que diz respeito à validade de critério, utilizou-se como critério de validade a medida de satisfação, conforme indicou a revisão bibliográfica
(TERBLANCHE; BOSHOFF, 2006; BONFIM et al., 2008) tendo-se verificado evidencias de validade, conforme indica o item 3.4.2;

- Para a avaliação da confiabilidade foram utilizados dois critérios: o coeficiente alpha de Cronbach, e a confiabilidade composta, também indicada como alternativa complementar para verificação de confiabilidade. Em todas as verificações, houve evidências de confiabilidade, conforme expõe o item 3.4.3;

Por fim, a escala ficou com quatro dimensões, das cinco inicialmente pensadas, com um total de 19 itens, com cada dimensão contendo entre 4 ou 5 itens de avaliação. Desta forma, tem-se disponível uma ferramenta que, em termos práticos, poderá servir para geração de informações para que gestores e empresários varejistas possam aprimorar o gerenciamento do ponto de venda.

Em uma perspectiva teórica, as informações contidas neste estudo complementam outros estudos já desenvolvidos com finalidades semelhantes (ver referencial teórico), contribuindo a partir de um contexto bem específico (ponto de venda varejista), mas que tem semelhanças com outros contextos de serviços. Adicionalmente, este estudo se insere no contexto geral da análise em marketing da avaliação dos clientes (especialmente na disciplina de comportamento do consumidor) trazendo um instrumento que pode contribuir para outros estudos nesta área.

Algumas das limitações merecem destaque, especialmente na amostra que gerou as análises confirmatórias, que foi coletada em apenas três segmentos (produtos infantis, produtos de informática, e moda praia), além de ter sido limitada a apenas uma cidade. Recomenda-se assim a aplicação dessa escala em outros contextos 
varejistas (supermercados, livrarias, farmácias...), o que deve servir como mecanismo de aprimoramento da própria escalam por meio de uma validação por segmento. Adicionalmente, e como forma de gerar uma validação de base geográfica (PONS; MOURALI; NYECK, 2006), recomendase a replicação dos procedimentos em outras cidades.

Por fim, recomenda-se que sejam inseridas outras dimensões de análise (tais como lealdade, comprometimento, envolvimento, valor...) para composição de modelos estruturais, o que viabilizaria condições de análise de validade nomológica da escala, e ainda o teste de hipóteses de relações entre os construtos no contexto varejista.

\section{Referências}

BAGOZZI, R. P.; EDWARDS, J. R. A general approach for representing constructs in organizational research. Organizational Research Methods, v. 1, n. 1, p. 45-87, 1998.

BAKER, J.; PARASURAMAN, A.; GREWAL, D.; VOSS, G. B. The influence of multiple store environment cues on perceived merchandise value and patronage intentions. Journal of Marketing, v. 66, p. 120-141, 2002.

BAKER, J. The role of the environment in marketing services: the consumer perspective. In. CZEPIEL, J. A.; CONGRAM, C. A.; SHANAHAN, J. (Eds). The services challenge: integrating for competitive advantage. Chicago: American Marketing Association, p. 79-84, 1987.

BAKER, J.; LEVY, M.; GREWAL, D. An experimental approach to making retail store environmental decisions. Journal of Retailing, v. 68, p. 445-61, 1992.
BITNER, M. J. Servicescapes: the impact of physical surroundings on customer and employees. Journal of Marketing, v. 56, p. 57-71, 1992.

BONFIM, D. G.; COSTA, F. J.; FREIRE, A. S.; MOREIRA, C. B. OLIVEIRA, D. M. A experiência no ponto de venda e sua influência sobre a satisfação, a lealdade e a confiança: uma análise no segmento varejista de livrarias. Revista Brasileira de Pesquisas de Marketing, Opinião e Mídia-PMKT, v. 1, n.1, p. 26-40, 2008.

BULTEZ, A.; NAERT, P. SHARP: shelf allocation for retailers' profit. Marketing Science, v. 7, n. 3, p. 211-31, 1988.

CHASE, R. B.; HAYES, R. H. Beefing up operations in service firms. Sloan Management Review, n. 32, p. 15-26, 1991.

CHURCHILL, G. A. A paradigm for developing better measures of marketing constructs, Journal of Marketing Research, v. 16, p. 64-73, 1979.

CORSTJENS, M.; DOYLE, P. A model for optimizing retail space allocations. Management Science, v. 27, n. 7, p. 822833, 1981.

COSTA, F. J.; BONFIM, D. G.; FREIRE, A. S.; LIMA, M. C. A experiência no ponto de venda e sua influência sobre a satisfação, a lealdade e a confiança de clientes de varejo. In. Encontro de Marketing, 3, Curitiba, 2008. Anais..., Curitiba: ANPAD, 2008.

COSTA, F. J.; SOARES, A. A. C. Uma análise da formação científica em cursos de graduação em administração: a perspectiva dos alunos. Revista de Gestão REGE, v. 15, n.1, p. 47-60, 2008.

COX, K. The effect of shelf space upon sales of branded products. Journal of Marketing Research, v. 7, n. 1, p. 55-58, 1970. 
CURHAN, R. The relationship between space and unit sales in supermarkets. Journal of Marketing Research, v. 9, p. 406-412, 1972

DABHOLKAR, P. A.; THORPE, D. I.; RENTZ, J. O. A measure of service quality for retail stores: scale development and validation. Journal of the Academy of Marketing Science, v. 24, n. 1, p. 3-16, 1996.

DARDEN, W. R.; ERDEM, O.; DARDEN, D. K. A comparison and test of three causal models of patronage intentions, In. DARDEN, W. R.; LUSCH, R. F. eds. Patronage Behavior and Retail Managemen. New York: North-Holland, 29-43, 1983.

DEVELLIS, R. F. Scale development: theory and applications. Newbury Park, CA: SAGE Publications, 1991.

DICKSON, J.; ALBAUM, G. A method for developing tailormade semantic differentials for specific marketing content areas. Journal of Marketing Research, v. 14, n. 1, p. 87-91, 1977.

DIXON, J.; BRIDSON, K.; EVANS, J.; MORRISON, M. An alternative perspective on relationship, loyalty and future store choice. International Review of Retail, Distribution and Consumer Research, v. 15, n. 4, p. 351-74, 2005.

DONOVAN, R.; ROSSITER, J. Store Atmosphere: An Environmental Psychology Approach. Journal of Retailing, v. 58, p. 34-57, 1982.

GREENLAND, S. J.; MCGOIDRICK, P. J. Atmospherics, attitudes and behavior: modeling the impact of designed Space. International Review of Retail, Distribution and Consumer Research, v. 4, p. 1-16, 1994.

GREENLEY, G. E.; SHIPLEY, D. D. An empirical overview of marketing by retailing organizations. Service Industries Journal, v. 8, n. 1. p. 49-66, 1988.

GREWAL, D.; SHARMA, A. The effect of salesforce behavior on customer satisfaction: an interactive framework. Journal of Personal Selling and Sales Management, v. 11, n. 3, p. 13-23, 1991.

GUTMAN, J.; ALDEN, S. D. Adolescents' cognitive structures of retail stores and fashion consumption: $\mathrm{A}$ means-end chain analysis of quality. In JACOBY, J.; OLSEN, J. (Eds.). Perceived quality: How consumers view stores and merchandise. Lexington, M.A.: Lexington Books, 1985.

HAIR J. F., ANDERSON, R. E., TATHAM, R. L., BLACK, W. C. Análise multivariada de dados. 5. ed. Porto Alegre: Bookman, 2005.

HASTY, R.; REARDON, J. Retail management. New York: McGraw-Hill, 1997.

HUMMEL, J. W.; SAVITT, R. Integrated customer service and retail strategy. International Journal of Retailing, v. 3, n. 2, p. 5-21, 1988.

KOELEMEIJER, K.; OPPEWAL, $\mathrm{H}$. Assessing the effects of assortment and ambience: a choice experimental approach. Journal of Retailing, v. 75, n. 3, p. 31945, 1999.

JARVIS, C. B.; MACKENZIE S. B.; PODSAKOFF, P. M. A critical review of construct indicators and measurement model misspecification in marketing and consumer research. Journal of Consumer Research, v. 30, n. 2, p. 199-218, 2003.

MALHOTRA, N. K. Marketing research: an applied orientation. 3. ed. New Jersey: Prentice-Hall, 1999.

MAZURSKY, D.; JACOBY, J. Forming impressions of merchandise and service 
quality. In JACOBY, J.; OLSEN, J. (Eds.). Perceived quality: How consumers view stores and merchandise. Lexington, M.A.: Lexington Books, 1985.

MCGOLDRICK, P. J.; GREENLAND, S.J. Retailing of financial services. London: McGraw-Hill, 1994.

NETEMEYER, R. G.; BEARDEN, W. O.; SHARMA, S. Scaling procedures: issues and applications. Thousand Oaks: Sage, 2003.

PARASURAMAN, A.; ZEITHAML, V. A.; BERRY, L. L. Servqual: a multipleitem scale for measuring consumer perceptions of service quality. Journal of Retailing, v. 64, p. 12-40, spring, 1988.

PARENTE, J. Varejo no Brasil. São Paulo: Atlas, 2000.

PONS, F.; MOURALI, M.; NYECK, S. Consumer orientation toward sporting events: Scale development and validation. Journal of Service Research, v. 8, n. 3, p. 276-287, 2006.

RIDGEWAY, N. M.; DAWSON, S. A.; BLOCH, P. H. Pleasure and arousal in the marketplace: interpersonal differences in approach-avoidance responses. Marketing Letters, v. 1, n. 2, p. 139-147, 1989.

SOARES, A. A. C. A influência do valor para o cliente e da satisfação no comportamento de boca-a-boca: uma análise no setor de serviços. 190f. Dissertação (Mestrado em Administração). Universidade Estadual do Ceará, 2008.

TERBLANCHE, N. S.; BOSHOFF, C. The relationship between a satisfactory in-store shopping experience and retailer loyalty. South African Journal of Business Management, v 37, n. 2, p. 33-43, 2006.

TERBLANCHE, N. S.; BOSHOFF, C. A generic instrument to measure customer satisfaction with the in-store shopping experience: some preliminary results'. In. Annual Conference of the European Marketing Academy, 31, Braga. Proceedings. Braga: EMAC, 2002.

TITUS, P. A.; EVERETT, P. B. The consumer retail search process: a conceptual model and research agenda. Journal of the Academy of Marketing Science, v. 23, n. 2, p. 106-19, 1995.

VIEIRA, V. A.; SLONGO, L. A. An inventory of the characteristics of the marketing scales created and tested in Brazil. Revista de Administração Mackenzie - RAM, v. 8, n. 4, p. 11-34, 2007.

WESTBROOK, R. A. Sources of consumer satisfaction with retail outlets. Journal of Retailing, v. 57, n. 3, p. 68-85, 1981

ZIMMER, M. R.; GOLDEN, L. L. Impressions of retail stores: a content analysis of consumer images. Journal of Retailing, v. 64, n. 3, p. 256-92, 1988. 